Pacific Journal of Mathematic 


\title{
ON CONTINUITY CONDITIONS FOR FUNCTIONS
}

\author{
Evelyn R. MCMILLAN
}

Suppose $f$ is a function from a topological space $X$ into a topological space $Y$. Many classical theorems of topology assert that from continuity of $f$ follow certain other properties. For example, if $f$ is continuous, then its point inverses must be closed (if $Y$ is a $T_{1}$ space) and compact subsets of $X$ must have compact images under $f$, that is, $f$ must be compact preserving. Also, $f$ must be connected, that is, connected subsets of $X$ have connected images. It is natural to ask whether some combination of these properties is equivalent to continuity.

In particular, our main result (Theorem 2) concerns compact preserving, connected functions. We give a proof of a result announced but inadequately proved by $E$. Halfar that such a function $f$ is continuous if $X$ is a locally connected Hausdorff space with property $K$ and $Y$ is a Hausdorff space. (A space $X$ has property $K$ if given that $x_{0}$ is a limit point of an infinite set $A \subset X$, then there is a compact set $K \subset A \cup\left\{x_{0}\right\}$ such that $x_{0}$ is a limit point of $K$.)

Examples are given in $\S 3$ to show that the conditions on the domain of $f$ cannot, in general, be omitted.

The result stated above generalizes theorems of other authors. Klee and Utz [11] proved that if $X$ is a locally connected metric space, then every compact preserving, connected function from $X$ into a metric space is continuous. Whyburn [19] showed that if the domain satisfies the first axiom of countability then "metric space" can be replaced at each occurrence by "Hausdorff space" in this result.

An important tool in our version of Halfar's theorem is the equivalence in Hausdorff spaces of property $K$ with the following "compactness condition": A space $X$ has property $E$ if given that $x_{0}$ is a limit point of an infinite set $A \subset X$, then there is an infinite sequence of distinct points $\left\{x_{i}\right\} \subset A$ such that $\left\{x_{i}\right\}$ converges to $x_{0}$. This surprising equivalence was pointed out to us by M. E. Rudin, whose slightly modified proof we include in $\S 2$. We are also indebted to her for the example in Remark 2 of $\S 3$.

We also consider spaces satisfying compactness conditions weaker than properties $E$ and $K$ : Property $\alpha$ spaces and spaces in which compactly closed sets are closed. A space $X$ has property $\alpha$ if given that $x_{0}$ is a limit point of an infinite set $A \subset X$, then there is a compact set $K \subset X$ such that $x_{0}$ is a limit point of $K \cap A$. A subset $A$ of a space $X$ is compactly closed if $A \cap K$ is closed in $K$ for each compact set $K \subset X$. A Hausdorff space with compactly closed sets closed is a 
$k$-space in the sense of Kelley [10]. It is easily seen that each of the properties of spaces in the following list implies the next: $T_{1}$ and satisfies the first axiom of countability, property $E$, property $K$, property $\alpha$, all compactly closed sets are closed. Section 2 contains examples to show that none of these implications is, in general, reversible.

As remarks in $\S 3$ and $\S 5$ indicate, there is some difficulty in pinpointing precisely those spaces $X$ for which the following proposition holds: every compact preserving connected function with domain $X$ is continuous. For instance, the example in Remark 2 of $\S 3$ shows that the proposition may fail if $X$ does not have compactly closed sets closed. On the other hand (see $\S 5$ ), neither property $K$ nor property $\alpha$ is a necessary condition on $X$ for the truth of the proposition. It seems reasonable to expect (because of an analogous result for compact preserving functions with closed point inverses) that the condition that $X$ have all compactly closed sets closed may be necessary.

Section 4 is concerned with compact preserving functions with closed point inverses. In [11] Klee and Utz showed that any such function between metric spaces is continuous. Extending this result to the case of such functions between Hausdorff spaces, various authors have shown that continuity follows if the domain is assumed to satisfy some compactness conditions: Halfar used property $K$, [8]; Fuller, property $\alpha$, [6]; Whyburn, compactly closed sets closed, [19]. In $\S 4$ we show that this last result of Whyburn cannot be improved, in the sense that a Hausdorff space $X$ has compactly closed sets closed if and only if it satisfies the following proposition: every compact preserving function with closed point inverses from $X$ into a Hausdorff space $Y$ is continuous.

For definitions not included in this paper, the reader is referred to $[10]$.

Some other papers, in addition to those mentioned above, which deal with the problem of finding conditions which imply continuity are [3], [7], [14], [15], [16], and [18].

2. Some compactness conditions. The main result of this section is that properties $E$ and $K$ are equivalent for Hausdorff spaces. First we give an example to show that, in general, property $E$ is stronger than property $K$. This and the other examples which follow show that no two of the compactness conditions defined in $\S 1$ are equivalent.

EXAmple 1. A $T_{1}$ space which has property $K$ but not property $E$ :

Let $X$ be the set of ordinals less than or equal to $\Omega$, where $\Omega$ is 
the first uncountable ordinal. Give $X$ the topology generated by the sets of the form

$$
S_{\alpha}=\{x \in X: x>\alpha\},
$$

for each $\alpha<\Omega$, together with those sets which are complements of finite sets. That is, this is a subbasis for the topology on $X$.

$X$ does not have property $E$, since $\Omega$ is a limit point of $X-\{\Omega\}$, but no sequence in $X-\{\Omega\}$ converges to $\Omega$. To see that $X$ does have property $K$, one need only note that every subset $A$ of $X$ is compact. For, if an open cover of $A$ is given, then the element of that cover which contains the first element of $A$ also contains all but a finite number of the remaining points of $A$.

ExAmple 2. A property $E$ space which does not satisfy the first axiom of countability:

Let $X=\bigcup_{n=1}^{\infty} L_{n}$, where $L_{n}$ is the line segment in the plane joining the points $(0,0)$ and $(1,1 / n)$. Let $X-\{(0,0)\}$ have the relative topology from the plane. A basis for the open sets about $(0,0)$ are sets of the form $\bigcup_{n=1}^{\infty} S_{n}$ where $S_{n} \subset L_{n}$ for each $n$ is a half-open interval with $(0,0)$ as the included end point. (See [8], p. 691.) Note that there is a countable basis at each point of $X$ except $(0,0)$. However, $X$ has property $E$, since if $(0,0)$ is a limit point of $A \subset X$ then for some $n,(0,0)$ is a limit point of $A \cap L_{n}$.

EXAMPLE 3. A compact Hausdorff space which has property $\alpha$ but not property $K$ :

Let $X$ be the set of ordinals less than or equal to the first uncountable ordinal, $\Omega$. Give $X$ the order topology. $X$ has property $\alpha$ since it is compact. However, $\Omega$ is a limit point of the set

$$
A=\{x+1: x \text { is a limit ordinal, } x<\Omega\},
$$

but no compact subset of $A \cup\{\Omega\}$ has $\Omega$ as a limit point.

Fuller in [6] indicates that he does not know whether property $\alpha$ and compactly closed sets closed are equivalent concepts. The example which follows shows that they are not.

EXAMPLE 4. A Hausdorff space in which all compactly closed sets are closed but which does not have property $\alpha$ : 


$$
X=\{(1 / n, 0)\}_{n=1}^{\infty} \cup\{(1 / n, 1 / m)\}_{n, m=1}^{\infty} \cup\{(0,0)\} .
$$

Let $X-\{(0,0)\}$ have the relative topology from the plane. A set $U$ containing $(0,0)$ will be open if $U$ contains all but a finite number of the points of the form $(1 / n, 0)$ and for each $n_{0}$ such that $\left(1 / n_{0}, 0\right) \in U$, $U$ contains all but a finite number of points of the form $\left(1 / n_{0}, 1 / m\right)$. (See [5], p. 51.)

$X$ does not have property $\alpha$, since $(0,0)$ is a limit point of

$$
\{(1 / n, 1 / m)\}_{n, m=1}^{\infty},
$$

but if $K$ is a compact set in $X$, then $K$ contains points of $\left\{\left(1 / n_{0}, 1 / m\right)\right\}_{m=1}^{\infty}$ for only finitely many $n_{0}$. Thus, $(0,0)$ is not a limit point of

$$
K \cap\{(1 / n, 1 / m)\}_{n, m=1}^{\infty} \text {. }
$$

To see that compactly closed sets in $X$ are closed, suppose $S \subset X$ is not closed. Then either $(0,0)$ or one of the points $(1 / n, 0)$ is a limit point of $S$ which is not in $S$.

If $\left(1 / n_{0}, 0\right)$ for some $n_{0}$ is a limit point of $S$ not in $S$, then $S$ must contain infinitely many points of the set $\left\{\left(1 / n_{0}, 1 / m\right)\right\}_{m=1}^{\infty}$. Let

$$
K=\left(S \cap\left\{\left(1 / n_{0}, 1 / m\right)\right\}_{m=1}^{\infty}\right) \cup\left\{\left(1 / n_{0}, 0\right)\right\} .
$$

Then $K$ is a compact set (a converging sequence plus its limit point), however, $K \cap S$ is not closed in $K$, since it does not contain the point $\left(1 / n_{0}, 0\right)$. Thus $S$ is not compactly closed.

If $S$ contains all of the points of the form $(1 / n, 0)$ which are limit points of $S$, then $(0,0)$ must be a limit point of $S$ which is not in $S$. It follows that $S$ must contain infinitely many points of $\{(1 / n, 0)\}_{n=1}^{\infty}$. For, suppose not. Let

$$
\left(1 / n_{1}, 0\right),\left(1 / n_{2}, 0\right), \cdots\left(1 / n_{k}, 0\right)
$$

be all of these points which are in $S$. Choose

$$
m>\max \left(n_{1}, n_{2}, \cdots, n_{k}\right),
$$

then no point $(1 / j, 0)$ with $j \geqq m$ is a limit point of $S$ or is in $S$. Thus, there is an open set about $(0,0)$ which contains no points of $S$. This is a contradiction, and it follows that $S$ contains infinitely many points of $\{(1 / n, 0)\}_{n=1}^{\infty}$. Let

$$
K=\left(S \cap\{(1 / n, 0)\}_{n=1}^{\infty}\right) \cup\{(0,0)\} .
$$

$K$ is a compact set, but $K \cap S$ is not closed, since it does not contain $(0,0)$.

Thus, in either case if $S$ is not closed, then $S$ is not compactly closed. It follows that the space $X$ has compactly closed sets closed. 
The following lemma, which is the key to showing that a Hausdorff space which has property $K$ also has property $E$, may be of independent interest. The proof of the lemma involves the Hausdorff Maximal Principle. (See [10], p. 32.)

Lemma. If $X$ is a Hausdorff space and $x_{0} \in X$ is a limit point of $X$, then there exists an infinite disjoint collection $\left\{U_{b}\right\}_{b \in B}$ of open subsets of $X-x_{0}$ such that $x_{0}$ is in the closure of their union, but $x_{0}$ is not in the closure of $U_{b}$ for any $b$.

Proof. Let $\mathscr{U}$ be the collection of all open subsets $U$ of $X-x_{0}$ such that $x_{0}$ is not in the closure of $U$. Since $X$ is a Hausdorff space, for each $y \in X-x_{0}$ there is an element $U_{y}$ of $\mathscr{Q}_{6}$ with $y \in U_{y}$. Note that $X-x_{0} \neq \varnothing$, since $x_{0}$ is a limit point of $X$. Thus, $\mathscr{C}$ is nonempty.

Let $\mathscr{A}$ consist of all subcollections of $\mathscr{C}$ whose elements are pairwise disjoint. $\mathscr{A}$ is partially ordered by inclusion. Let $\mathscr{T}$ be any tower of elements of $\mathscr{A}$. Then by the Hausdorff Maximal Principle there is a maximal tower $\mathscr{C}$ containing $\mathscr{N}$.

Let $\mathscr{C l}=\left\{A_{c}\right\}_{c \in C}$ and $\left\{U_{b}\right\}_{b \in B}=\bigcup_{c \in C} A_{c}$. We claim that $x_{0}$ is a limit point of $\bigcup_{b \in B} U_{b}$. Suppose not. Choose an open set $W$ about $x_{0}$ such that $W$ contains no points of $\bigcup_{b \in B} U_{b}$. Choose $y \in W \cap\left(X-x_{0}\right)$. Let $V_{1}$ and $V_{2}$ be disjoint open sets containing $y$ and $x_{0}$ respectively. Then $U_{y}=V_{1} \cap W$ is in $\mathscr{U}$, is not in $\left\{U_{b}\right\}_{b \in B}$, but is disjoint from each $U_{b}$. This is a contradiction since $\mathscr{C}$ was a maximal tower.

Thus, $x_{0}$ is a limit point of $\mathbf{U}_{b \in B} U_{b}$. Note that $x_{0}$ is not in the closure of $U_{b}$ for each $b$ since each $U_{b}$ is in $\mathscr{U}$.

Theorem 1 (M. E. Rudin). If $X$ is a Hausdorff space, then $X$ has property $E$ if and only if $X$ has property $K .^{1}$

Proof. As indicated in $\S 1$, it is obvious that if $X$ has property $E$, then $X$ has property $K$.

Suppose $X$ has property $K$. Let $A$ be an infinite subset of $X$ with $x_{0} \in X$ a limit point of $A . A \cup\left\{x_{0}\right\}$ is a Hausdorff space. Applying the lemma, there is a collection $\left\{U_{b}\right\}_{b \in B}$ of pairwise disjoint subsets of $A-\left\{x_{0}\right\}$, such that each $U_{b}$ is open in $A-\left\{x_{0}\right\}, x_{0}$ is a limit point of $\mathrm{U}_{b \in B} U_{b}$, but $x_{0}$ is not in the closure of $U_{b}$ for any $b$.

Choose a compact set $K$ such that $x_{0}$ is a limit point of $K$ and

$$
K \subset\left(\bigcup_{b=B} U_{b}\right) \cup\left\{x_{0}\right\} .
$$

$K$ contains points of infinitely many $U_{b}$, since otherwise $x_{0}$ would be a limit point of a finite collection $\left\{U_{i}\right\}_{i=1}^{n}$ and hence, of some one $U_{i}$,

1 Added in proof: Another proof may be found in Theorem 1 of A. Arhangelskij, $A$ characterization of very $k$-spaces, Czech. Math. J. 18 (93) (1968), 392-395. 
contrary to the fact that $x_{0}$ is not in the closure of any $U_{b}$. Let $\left\{b_{i}\right\}_{i=1}^{\infty}$ be any infinite sequence of indices such that $K \cap U_{b_{i}} \neq \varnothing$ for each $i$. Choose $x_{i}$ in $U_{b_{i}} \cap K, i=1,2,3, \cdots$. We claim that $\left\{x_{i}\right\}$ converges to $x_{0}$. Suppose not. Choose an open set $W$ about $x_{0}$ with infinitely many points $x_{i}$ in $X-W . K$ is covered by $W$ together with $\left\{V_{b}\right\}_{b \in B}$ where $V_{b}$ is open in $X$ and $U_{b}=V_{b} \cap\left(A-\left\{x_{0}\right\}\right)$. This cover connot be reduced to a finite subcover since infinitely many sets $V_{b}$ are required to cover the points in $(X-W) \cap\left\{x_{i}\right\}$. This is a contradiction since $K$ is compact. Thus $\left\{x_{i}\right\}$ converges to $x_{0}$.

Thus $X$ has property $E$, and the proof is complete.

3. Halfar's theorem. E. Halfar [8, Th. 6, p. 690-691] purported to prove the following theorem: If $X$ is a locally connected Hausdorff space with property $K$ and $f$ is a compact preserving, connected function from $X$ into a Hausdorff space $Y$, then $f$ is continuous. However, there appear to be gaps in his proof. The main objection is in the second paragraph. By Theorem 5 of [8] it suffices for him to show that $f$ has closed point inverses. Thus, he supposes that there is a point $x_{0}$ such that

$$
x_{0} \in \overline{f^{-1}\left(y_{0}\right)}-f^{-1}\left(y_{0}\right) .
$$

He claims to construct a compact set $K$ with $x_{0}$ as a limit point and $f(K)$ infinite. To do this he does not make full use of the connectedness of the function-only that connected neighborhoods of $x_{0}$ have connected images. The following example shows that this is not sufficient in trying to obtain such a compact set. The function below on a property $K$ space is neither continuous nor connected, but connected neighborhoods of $x_{0}$ do have connected images.

Let $X$ be the closed triangular region in the plane bounded by the triangle $a b x_{0}$, where $a=(0,0), b=(1,0)$, and $x_{0}=(1 / 2,1)$. Open sets about $p \in X-\left\{x_{0}\right\}$ are those subsets of $X-\left\{x_{0}\right\}$ which as subsets of the line segment through $p$ joining $x_{0}$ to the base are open in the relative Euclidean topology for this line segment. An open neighborhood of $x_{0}$ is obtained by choosing on each line segment, between $x_{0}$ and the base of the triangle, a point $p_{a} \neq x_{0}$ and taking the union of the half open segments $\left(p_{a}, x_{0}\right]$.

Let $Y$ be the unit interval, $[0,1]$, with the usual topology. Define $f: X \rightarrow Y$ by $f\left(x_{0}\right)=1$ and on $X-\left\{x_{0}\right\}, f$ is projection from $x_{0}$.

$X$ is locally connected and the image of every neighborhood of $x_{0}$ is $Y$ and hence is connected. Thus, in particular, connected neighborhoods in $X$ have connected images. However, every compact set $K \subset X$ with $x_{0}$ as limit point has a finite image, since $K$ must be contained in only finitely many rays. 
A second objection to Halfar's argument is that assuming he obtains $K$ with $f(K)$ infinite, it is not clear why $f$ is one-to-one on $K$. $\mathrm{He}$ needs this to be true at several points in the remainder of his proof.

We show below that the theorem Halfar stated is in fact true. First, we give some lemmas. Lemma 1 states that on a property $E$ space continuity of a function is equivalent to sequential continuity. The proof is straightforward, and we omit it. Lemma 2 shows that a compact preserving function into a Hausdorff space already has a certain amount of sequential continuity. Lemma 3 is used only once in the proof of the theorem. It is stated separately to emphasize that only the connectedness hypotheses are needed for that part of the proof.

Lemma 1. If $X$ has property $E$ and $f: X \rightarrow Y$ is a function, then $f$ is continuous at $x_{0} \in X$ if and only if for every sequence $\left\{x_{i}\right\}$ which converges to $x_{0}$, it is true that $\left\{f\left(x_{i}\right)\right\}$ converges to $f\left(x_{0}\right)$.

LEMMA 2. If $f: X \rightarrow Y$ is a compact preserving function, $Y$ is a Hausdorff space, $\left\{x_{i}\right\} \subset X$ converges to $x_{0} \in X$ and $f\left(x_{i}\right) \neq f\left(x_{j}\right)$ for $i \neq j$, then $\left\{f\left(x_{i}\right)\right\}$ converges to $f\left(x_{0}\right)$.

Proof. Let $\left\{x_{i}\right\}$ and $x_{0}$ be as in the hypothesis. Suppose $\left\{f\left(x_{i}\right)\right\}$ does not converge to $f\left(x_{0}\right)$. Let $V$ be an open set about $f\left(x_{0}\right)$ whose complement contains infinitely many points of the set $\left\{f\left(x_{i}\right)\right\}$. Let $\left\{y_{i}\right\}$ be a subsequence of $\left\{x_{i}\right\}$ with $\left\{f\left(y_{i}\right)\right\}$ infinite and

$$
\left\{f\left(y_{i}\right)\right\} \subset Y-V,
$$

Now $\left\{y_{i}\right\} \cup\left\{x_{0}\right\}$ is compact. Hence,

$$
\left\{f\left(y_{i}\right)\right\}=f\left(\left\{y_{i}\right\} \cup\left\{x_{0}\right\}\right) \cap(Y-V)
$$

is compact. Thus some $f\left(y_{j}\right)$ is a limit point of $\left\{f\left(y_{i}\right)\right\}$. Let $\left\{z_{i}\right\}$ be the subsequence of all elements of $\left\{y_{i}\right\}$ with $f\left(z_{i}\right) \neq f\left(y_{j}\right)$. Now $\left\{z_{i}\right\} \cup$ $\left\{x_{0}\right\}$ is compact, but

$$
\left\{f\left(z_{i}\right)\right\}=f\left(\left\{z_{i}\right\} \cup\left\{x_{0}\right\}\right) \cap(Y-V)
$$

is not compact, since it is not closed. This is a contradiction since $f\left(\left\{z_{i}\right\} \cup\left\{x_{0}\right\}\right)$ is compact and $Y-V$ is closed.

Thus, $\left\{f\left(x_{i}\right)\right\}$ must converge to $f\left(x_{0}\right)$.

LemMa 3. Suppose $f$ is a connected function from a locally connected space $X$ into a $T_{1}$ space $Y$. Let $x \in X$ be a limit point of $f^{-1}(F)$, where $F \subset Y$ is closed. If $x$ has an open neighborhood $U$ such that $U \cap f^{-1}(F)$ is open in $X$, then $x \in f^{-1}(F)$. 
Proof. Since $X$ is locally connected, we may assume that $U$ is connected. Suppose $x$ is not in $f^{-1}(F)$. We claim that $U \cap f^{-1}(F)$ is not connected. If it were, then it together with its limit point $x$ would be a connected set whose image is not connected.

Consider a component $C$ of $U \cap f^{-1}(F) . \quad C$ is open since $X$ is locally connected. Since $U$ is connected, it cannot be true that

$$
(\bar{C}-C) \cap U=\varnothing \text {. }
$$

Choose $t \in(\bar{C}-C) \cap U$. Since $t$ is not in $f^{-1}(F)$, the connected set $C \cup\{t\}$ does not have a connected image. ${ }^{2}$ This contradicts the hypothesis that $f$ is a connected function.

Thus, $x$ is in $f^{-1}(F)$.

We are now ready to prove the theorem which Halfar stated. Note that we state it with property $E$ replacing property $K$. However, in view of the equivalence of these two properties for Hausdorff spaces, Theorem 2 below is equivalent to Halfar's statement.

THEOREM 2. If $X$ is a locally connected, Hausdorff space with property $E$, and $f$ is a compact preserving, connected function from $X$ into a Hausdorff space $Y$, then $f$ is continuous.

Proof. Suppose $f$ is not continuous at $x \in X$. Then by Lemma 1 there exists an infinite sequence $\left\{x_{i}\right\} \subset X$ which converges to $x$, but $\left\{f\left(x_{i}\right)\right\}$ does not converge to $f(x)$. By Lemma 2 we may assume that for some $y \in Y, y \neq f(x), f\left(x_{i}\right)=y$ for infinitely many $i$. Thus

$$
x \in \overline{f^{-1}(y)}-f^{-1}(y) .
$$

Choose disjoint open sets $U$ and $V$ about $f(x)$ and $y$, respectively.

Decompose $f^{-1}(y)$ into three mutually exclusive sets $Y_{1}, Y_{2}$, and $Y_{3}$. Let $Y_{1}$ consist of the interior points of $f^{-1}(y)$. If $z$ is in $f^{-1}(y)$ but is not an interior point, then $z$ must be a limit point of $X-f^{-1}(y)$. Thus, by property $E$ some sequence in $X-f^{-1}(y)$ converges to $z$. Let

$$
Y_{2}=\left\{\begin{array}{ll}
z \in f^{-1}(y): & z \notin Y_{1} \text { and there exists an infinite sequence } \\
& \left\{z_{i}\right\} \text { of points from distinct point inverses } \\
& f^{-1}\left(w_{i}\right) \text { which converges to } z
\end{array}\right\}
$$

and

2 This argument is essentially that used to show that components of the inverse of a closed set are closed under a connected function, if the range is $T_{1}$. (See Hamilton, Fixed points for certain noncontinuous transformations, Proc. Amer. Math. Soc. 8 (1957), 750-756.) The author wishes to thank the referee for indicating this stronger result for connected functions and pointing out that it could be used to give a direct proof of Lemma 3. 


$$
Y_{3}=\left\{\begin{array}{ll}
z \in f^{-1}(y): \begin{array}{l}
z \notin Y_{1} \text { and for each sequence }\left\{z_{i}\right\} \text { in } X-f^{-1}(y) \\
\text { which converges to } z,\left\{f\left(z_{i}\right)\right\} \text { is finite }
\end{array}
\end{array}\right\} .
$$

Since $x$ is a limit point of $f^{-1}(y)$, it must be a limit point of at least one of the subsets $Y_{i}, i=1,2$, or 3 .

(1) Suppose $x \in \bar{Y}_{2}$, then there is an infinite sequence $\left\{z_{i}\right\} \subset Y_{2}$ which converges to $x$. For each $z_{i}$ there is an infinite sequence $\left\{z_{i j}\right\}$ which converges to $z_{i}$ with

$$
z_{i j} \in f^{-1}\left(w_{i j}\right), w_{i j_{1}} \neq w_{i j_{2}}, \quad \text { if } j_{1} \neq j_{2} .
$$

Since $f\left(z_{i}\right)=y$ for each $i$, each of the sequences $\left\{w_{i j}\right\}_{j=1}^{\infty}$ must converge to $y$, by Lemma 2. Thus, we may assume that all of the points $w_{i j}$ are in the open set $V$ about $y$. We show that the sequences $\left\{z_{i j}\right\}_{i, j=1}^{\infty}$ can be chosen so that a point inverse $f^{-1}\left(w_{i j}\right)$ is represented only finitely many times.

Consider $z_{11} \in f^{-1}\left(w_{11}\right)$. If $z_{i j} \in f^{-1}\left(w_{11}\right)$ for $i>1$, then omit that $z_{i j}$ from that sequence. Consider $z_{12} \in f^{-1}\left(w_{12}\right)$. If $f^{-1}\left(w_{12}\right)$ is represented in any sequence after the second, omit each such representative. Thus, for each $n$ consider $z_{1 n} \in f^{-1}\left(w_{1 n}\right)$. If $f^{-1}\left(w_{1 n}\right)$ is represented in any sequence after the $n$ th, omit each such representative. Now each point inverse represented in the first sequence is represented in only finitely many of the remaining sequences.

Consider the $j$ th sequence and the first element of it still remaining, $z_{j m} \in f^{-1}\left(w_{j m}\right)$. If $f^{-1}\left(w_{j m}\right)$ is represented in any sequence after the $j$ th, then omit each such representative. Consider the next remaining element in the $j$ th sequence, $z_{j m^{\prime}} \in f^{-1}\left(w_{j m^{\prime}}\right)$. If $f^{-1}\left(w_{j m^{\prime}}\right)$ is represented in any sequence after the $(j+1)$ st, then omit each such representative. Continue in this manner for the rest of the elements of the $j$ th sequence.

Now, if $z_{i j} \in f^{-1}\left(w_{i j}\right)$ remains at the end of this process, then the point inverse $f^{-1}\left(w_{i j}\right)$ is represented in at most $(i+j)$ sequences. On the other hand, at most $i(i-1) / 2$ elements have been omitted from the $i$ th sequence.

Denote the countably many sequences remaining $\left\{z_{i j}\right\}$, then $x$ is a limit point of $\left\{z_{i j}\right\}$. Hence some sequence $\left\{s_{i}\right\} \subset\left\{z_{i j}\right\}$ converges to $x$. Now $\left\{f\left(s_{i}\right)\right\}$ is infinite, since by the process above a given point inverse is represented only finitely many times in $\left\{z_{i j}\right\}$. However, $f\left(s_{i}\right)$ is contained in the open set $V$ about $y$ for each $i$. Thus $\left\{f\left(s_{i}\right)\right\}$ does not converge to $f(x)$. This contradicts Lemma 2, and we must have $x \notin \bar{Y}_{2}$.

(2) We will show in this part that $x$ cannot be a limit point of $Y_{3}$. First, we demonstrate a useful property of the set $Y_{3}$. If $z \in Y_{3}$, then $z$ is an interior point of the set

$$
W_{z}=\bigcup\left\{f^{-1}(w): z \in \overline{f^{-1}(w)}\right\} .
$$


Note that $f^{-1}(y) \subset W_{z}$ since $z \in f^{-1}(y)$. Thus, $z$ is in $W_{z}$. It follows from the definition of $Y_{3}$ that $z$ cannot be a limit point of $X-W_{z}$. Thus $z$ has an open neighborhood $U_{z}^{\prime}$ with $U_{z}^{\prime}$ contained in $W_{z}$. Note that if $U_{z}^{\prime} \cap f^{-1}(q) \neq \varnothing$ for some $q$, then $f^{-1}(q) \subset W_{z}$, that is, $z$ is in the closure of $f^{-1}(q)$. We will also always choose $U_{z}^{\prime}$ to be connected in what follows.

Suppose $x \in \bar{Y}_{3}$, then there is an infinite sequence $\left\{z_{i}\right\} \subset Y_{3}$ which converges to $x$. For each $i$, there is a $w_{i}$ in $Y$ such that $z_{i}$ is the limit of a sequence in $f^{-1}\left(w_{i}\right)$. We show that the $w_{i}$ 's can be chosen distinct from one another.

There is some $w_{1}$ in $Y$ different from $f(x)$ and from $y$ such that $z_{1}$ is a limit point of $f^{-1}\left(w_{1}\right)$. For otherwise, the image of the connected neighborhood $U_{z_{1}}^{\prime}$ about $z_{1}$, whose existence was demonstrated above would be $\{f(x), y\}$, which is not connected. Let $K_{1}$ and $K_{0}$ be the closures of disjoint open sets containing $w_{1}$ and $f(x)$, respectively, with $y$ not in $K_{1} \cup K_{0}$. Consider $U_{z_{2}}^{\prime}$, the connected open neighborhood of $z_{2}$ whose existence was demonstrated above. $f\left(U_{z_{2}}^{\prime}\right)$ contains $y$ and some $w \neq y$. Thus, it must contain some point $w_{2}$ not in the set $K_{1} \cup K_{0} \cup\{y\}$, since otherwise, it would not be connected. Then $z_{2}$ is a limit point of $f^{-1}\left(w_{2}\right)$. Choose $K_{2}$ to be a closed neighborhood of $w_{2}$ with $y$ not in $K_{2}$. As for $z_{2}$, we can choose $w_{3}$ in

$$
Y-\left(K_{0} \cup K_{1} \cup K_{2} \cup\{y\}\right)
$$

with $z_{3}$ a limit point of $f^{-1}\left(w_{3}\right)$.

Continuing in this way we get each $z_{i}$ the limit of a sequence from a point inverse $f^{-1}\left(w_{i}\right)$ with $w_{i} \neq w_{j}$, if $i \neq j$. Choose a sequence $\left\{t_{i}\right\}$ in the union of these sequences such that $\left\{t_{i}\right\}$ converges to $x$. Now $\left\{f\left(t_{i}\right)\right\}$ is infinite, however,

$$
\left\{f\left(t_{i}\right)\right\} \subset Y-K_{0} \quad \text { and } f(x) \in \operatorname{Int} K_{0} .
$$

Thus, $\left\{f\left(t_{i}\right)\right\}$ does not converge to $f(x)$. This contradicts Lemma 2, and we must have $x \notin \bar{Y}_{3}$.

(3) Since $x$ is not a limit point of $Y_{2} \cup Y_{3}$, there is an open neighborhood $U$ of $x$ with

$$
U \cap\left(Y_{2} \cup Y_{3}\right)=\varnothing \text {. }
$$

That is, $U \cap f^{-1}(y)$ is an open set. From Lemma 3 it follows that $x \in f^{-1}(y)$, contrary to the original assumption.

It follows that $f$ is continuous at $x$.

REMARK 1. If the hypothesis that $X$ is locally connected is removed from the statement of Theorem 2, the resulting statement is not true in general. Klee and Utz [11] prove that if $X$ is a metric 
space which is not locally connected, then $X$ admits a real-valued compact preserving and connected function which is not continuous. The following example is suggested by their argument.

$A$ compact preserving, connected, but not continuous function $f$ from a non-locally connected metric space onto the interval [0,1/4]:

Let

$$
X=\{(x, \sin 1 / x): 0<x \leqq 1\} \cup\{(0,0)\}
$$

with the relative topology from the plane. Let $0=(0,0)$. Define $f$ : $X \rightarrow[0,1 / 4]$ by

$$
f(p)= \begin{cases}0 & \text { if } p=0 \text { or } d(p, 0) \geqq 1 / 2 \\ 1 / 2-d(p, 0) & \text { if } 1 / 4<d(p, 0)<1 / 2 \\ 1 / 4 & \text { if } 0<d(p, 0) \leqq 1 / 4\end{cases}
$$

It is easily seen that $f$ is continuous on $X-0$ but not at 0 . However, $f$ is compact preserving, since if $A \subset X$ is compact, then so is the set

$$
Q=A \cap\left(X-N_{1 / 4}(0)\right) \text {. }
$$

Thus, since $f$ is continuous on $X-0, f(Q)$ is compact. Since the image of the remainder of $A$ can be at most the two numbers $1 / 4$ and 0 , it follows that $f(A)$ is compact.

Also, $f$ is connected. Let $C \subset X$ be a connected set. If 0 is not in $C$, then $f(C)$ is connected, since $f$ is continuous on $X-0$. If $0 \in C$ and $C-0 \neq \varnothing$, then $f(C)=[0,1 / 4]$ since $C$ must contain points at a distance $r$ from 0 for each $0 \leqq r<1$.

REMARK 2. If the hypothesis that $X$ has property $E$ is removed from the statement of Theorem 2 the resulting statement is not true in general.

A compact preserving, connected, but not continuous function $f$ from a locally connected Hausdorff space $X$ onto the unit interval:

Let

$$
X=\{(\alpha, x): \alpha \text { is a countable ordinal and } 0 \leqq x<1\} \cup\{\Omega\} .
$$

The topology on $X$ has basis consisting of the following sets: for each $(\alpha, x) \in X$ and $\varepsilon>0$

$$
U_{\varepsilon}(\alpha, x)=\{(\alpha, y) \in X: x-\varepsilon<y<x+\varepsilon\}
$$

and for each $(\alpha, x) \in X$

$$
V(\alpha, x)=\{(\beta, y) \in X: \beta>\alpha, 0 \leqq y<1\} \cup\{(\delta, y) \in X: y>x\} \cup\{\Omega\} .
$$

Define $f: X \rightarrow[0,1]$ by $f(\alpha, x)=x$ and $f(\Omega)=1$. 
$f$ is continuous on $X-\{\Omega\}$, but it fails to be continuous at $\Omega$, since every open set about $\Omega$ has $[0,1]$ as its image. However, $f$ is compact preserving, since any compact set $K$ in $X$ can contain points in only finitely many sets $\alpha \times[0,1)$, and the intersection of $K$ with each $(\alpha \times[0,1)) \cup\{\Omega\}$ must be compact. Hence, $f(K)$ is the union of finitely many compact sets and thus is compact.

Also, $f$ is connected. Let $C \subset X$ be a connected set. If $\Omega$ is not in $C$, then $C \subset \alpha \times[0,1)$ for some $\alpha$. Thus, since $f$ is continuous on each $\alpha \times[0,1), f(C)$ is connected. If $\Omega \in C$ and $C$ is nondegenerate, then each set

$$
C_{\alpha}=C \cap(\alpha \times[0,1)),
$$

which is nonempty, has $\Omega$ as a limit point and is connected. Thus, $f\left(C_{\alpha}\right)=[x, 1)$ or $f\left(C_{\alpha}\right)=(x, 1)$ for some $x$ with $0 \leqq x<1$. Hence, $f(C)$ is connected, since it is the union of a collection of connected sets each of which has $f(\Omega)=1$ as a limit point.

$X$ is locally connected, since basic open sets are connected. However, $X$ not only does not have property $E$, but in fact, it does not have compactly closed sets closed. Property $E$ fails since $\Omega$ is a limit point of the set

$$
A=\{(\alpha, 0): \alpha<\Omega\},
$$

but no sequence in $A$ has $\Omega$ as limit point. Also, $A$ is compactly closed, since $A \cap K$ for any compact set $K \subset X$ is a finite set, but $A$ is not closed.

4. A characterization of spaces with compactly closed sets closed. Whyburn has shown that any compact preserving function with closed point inverses from a Hausdorff space with compactly closed sets closed into a Hausdorff space is continuous. We show in Theorem 3 below that it is necessary that the domain (if it is a $T_{1}$ space) have compactly closed sets closed in order that every such function on it be continuous.

Given a space $X$ we define an associated space $X_{k}$ (see [17]) which has the same compact subsets and has compactly closed sets closed. Let $X_{k}$ be the space with the same set of points as $X$ and with topology defined as follows: $U \subset X_{k}$ is open in $X_{k}$ if $U \cap K$ is open in $K$ (using the relative topology from $X$ on $K$ ) for each compact subset $K$ of $X$.

Note that the topology on $X_{k}$ contains the topology on $X$. For this reason if $K$ is a compact subset of $X_{k}$ then it is compact as a subset of $X$. The converse is also true. Suppose $K$ is a compact subset of $X$. Since the relative topologies $K$ inherits from $X$ and $X_{k}$ are the same, $K$ must be compact as a subset of $X_{k}$. Thus, $X$ and $X_{k}$ have the same compact subsets. Also, $X$ and $X_{k}$ have the same 
compactly closed sets, again, because a compact set $K$ has the same relative topology in the two spaces.

We claim that if $S$ is compactly closed in $X_{k}$ then it is closed in $X_{k}$. Equivalently, we show that $U=X_{k}-S$ is open in $X_{k}$. If $K$ is a compact set, then

$$
U \cap K=K-(S \cap K) .
$$

Since $S$ is compactly closed, $S \cap K$ is closed in $K$. Thus, $U \cap K$ is open in $K$ and $U$ is open in $X_{k}$.

THEOREM 3. If $X$ is a $T_{1}$ space containing a compactly closed set which is not closed, then there is a function with $X$ as domain which is compact preserving and has closed point inverses but is not continuous.

Proof. Let $X_{k}$ be the space associated with $X$ which was defined above. Let $f$ be the identity transformation from $X$ onto $X_{k}$. We show that if $S$ is any subset of $X$ which is compactly closed and $p$ is a point in $\bar{S}-S$, then $f$ is not continuous at $p$. Let $U=X_{k}-S$. Since $S$ is compactly closed in $X$, it is compactly closed in $X_{k}$. Thus. $S$ is closed in $X_{k}$ and hence, $U$ is open in $X_{k}$. Note that $p=f(p)$ is in $U$. However, every neighborhood of $p$ which is open in $X$ contains points of $S$ and hence, is not contained in $U$. Thus $f$ is not continuous at $p$.

Since $X$ and $X_{k}$ have the same compact sets, $f$ is compact preserving. Since $X$ is a $T_{1}$ space, $f$ has closed point inverses. This completes the proof.

CoRollary. If $X$ is a Hausdorff space then $X$ has compactly closed sets closed if and only if every compact preserving function with closed point inverses from $X$ into a Hausdorff space $Y$ is continuous.

Proof. We need only remark that if $X$ is a Hausdorff space then so is the associated space $X_{k}$.

5. Extending Halfar's theorem. Let us say that a locally connected Hausdorff space $X$ has property $(*)$ if each compact preserving, connected function from $X$ into a Hausdorff space is continuous. We have shown that each of properties $E$ and $K$ implies property $(*)$. Is there a weaker condition than property $K$ which implies property (*), that is, can the compactness condition on $X$ in Theorem 2 be weakened? Conversely, if a locally connected space has property (*), does it necessarily satisfy one of the compactness conditions? 
With regard to the first question, it seems likely that if a locally connected, Hausdorff space $X$ has property $\alpha$ and if $X-\{p\}$ has property $E$ for some point $p$ in $X$, then $X$ has property $(*)$. This is suggested by the case in which $X$ is the long line with the point $\Omega$ added (see [9], p. 55). The proof that $X$ does have property $(*)$ is contained in [13]. Note that this example also shows that neither properties $E$ nor $K$ is a necessary condition for a locally connected Hausdorff space to have property $(*)$.

Property $(*)$ also does not imply property $\alpha$ as the following example, in which $X$ is a modification of Example 4 of $\S 2$, shows.

EXAMPle 4'. A locally connected, Hausdorff space which does not have property $\alpha$, but on which every compact preserving, connected function into a Hausdorff space is continuous:

Let

$X=\{(x, 0): 0 \leqq x \leqq 1\} \cup\{(1 / n, y): 0 \leqq y \leqq 1$ and $n$ is a positive integer $\}$.

Let $X-\{(0,0)\}$ have the relative topology from the plane. A basic open set about $(0,0)$ is of the form

$$
\{(x, 0): 0 \leqq x<y\} \cup \bigcup_{1 / n<y} 1 / n \times\left[0, y_{n}\right)
$$

where $0<y \leqq 1$ and $0<y_{n} \leqq 1$ for each $n$.

An argument similar to that given in the discussion of Example $4, \S 2$ will show that $X$ does not have property $\alpha$ but does have compactly closed sets closed. $X$ is locally connected, since basic open sets are connected.

To see that every compact preserving connected function $f$ from $X$ into a Hausdorff space is continuous, first note that, by Theorem 2, such an $f$ is continuous on $X-\{(0,0)\}$, since $X-\{(0,0)\}$ has property $E$. The subspace

$$
\{(x, 0): 0 \leqq x \leqq 1\}
$$

of $X$ has property $E$. Thus, if $W$ is an open set about $f(0,0)$, then there is a set

$$
A_{y}=\{(x, 0): 0 \leqq x<y\} \text { such that } f\left(A_{y}\right) \subset W .
$$

If $1 / n<y$, then $f$ is continuous at $(1 / n, 0)$. Hence for each such $1 / n$ there is some $y_{n}$ between 0 and 1 so that

$$
f\left(1 / n \times\left[0, y_{n}\right)\right) \subset W .
$$

Thus, we have an open set $U$ about $(0,0)$ 


$$
U=A_{y} \cup \bigcup_{1 / n<y} 1 / n \times\left[0, y_{n}\right),
$$

such that $f(U) \subset W$. Consequently, $f$ is continuous at $(0,0)$.

Perhaps, property $(*)$ does imply that all compactly closed sets are closed. This conjecture is suggested by the analogous result (Theorem 3) for compact preserving functions with closed point inverses. Also the space $X$ in Remark 2 of $\S 3$, which fails to have property (*), does not have compactly closed sets closed.

We do have the following result which extends Theorem 2 to multifunctions. See Whyburn's paper [19] for all definitions. This result also extends Corollary $\mathrm{C}_{6}$ of [19]. The proof is essentially the same as that for Theorem 2.

THEOREM 4. If $X$ is a locally connected Hausdorff space with property $E$, and $f$ is a compact preserving, connected multifunction with nonmingled values from $X$ into a Hausdorff space $Y$, then $f$ is upper semicontinuous.

\section{REFERENCES}

1. Edwin Duda, Reflexive compact mappings, Proc. Amer. Math. Soc. 17 (1966), 688-693.

2. R. M. Dudley, On sequential convergence, Trans. Amer. Math. Soc. 112 (1964), 483-507.

3. Ky Fan and R. A. Struble, Continuity in terms of connectedness, Nederl. Akad. Wetensch. Proc., Ser. A 57 (1954), 161-164.

4. S. P. Franklin, Spaces in which sequences suffice, Fund. Math. 57 (1965), 107-116.

5. _ـ Spaces in which sequences suffice, II, Fund. Math. 61 (1968), 51-56.

6. R. V. Fuller, Relations among continuous and various noncontinuous functions, Pacific J. Math. 25 (1968), 495-509.

7. Edwin Halfar, Compact mappings, Proc. Amer. Math. Soc. 8 (1957), 828-830.

8. Conditions implying continuity of functions, Proc. Amer. Math. Soc. 11 (1960), 688-691.

9. J. G. Hocking and G. S. Young, Topology, Addison-Wesley, Reading, Mass. 1961.

10. J. L. Kelley, General topology, D. Van Nostrand Co., Princeton, N. J., 1955.

11. V. L. Klee and W. R. Utz, Some remarks on continuous transformations, Proc. Amer. Math. Soc. 5 (1954), 182-184.

12. W. E. Malbon, Invariants for quasi-compact mappings, University of Virginia Dissertation, 1955.

13. E. R. McMillan, On continuity conditions for functions, University of Virginia Dissertation, 1968.

14. W. J. Pervin and Norman Levine, Connected mappings of Hausdorff spaces, Proc. Amer. Math. Soc. 9 (1958), 488-495.

15. C. H. Rowe, Note on a pair of properties which characterize continuous functions. Bull. Amer. Math. Soc. 32 (1926), 285-287.

16. T. Tanaka, On a family of connected subsets and topology of spaces, J. Math. Soc. Japan 7 (1955), 389-393.

17. Seth Warner, The topology of compact convergence on continuous function spaces, Duke Math. J. 25 (1958), 265-282.

18. G. T. Whyburn, The most general closed point set over which continuous function may be defined by certain properties, Bull. Amer. Math. Soc. 33 (1927), 185-188. 
19. Continuity of multifunctions, Proc. Nat. Acad. Sci., U.S.A. 54 (1965), $1494-1501$.

20. Directed families of sets and closedness of functions, Proc. Nat. Acad. Sci., U.S.A. 54 (1965), 688-692.

Received January 22, 1969. This paper contains the substance of the author's thesis at the University of Virginia, 1968, prepared under the direction of Professor G. T. Whyburn. Support was provided during the academic year 1965-1966 by an NSF Cooperative Fellowship.

The UNiversity of Virginia

The UNIVERsity OF Wisconsin 


\section{PACIFIC JOURNAL OF MATHEMATICS}

\section{EDITORS}

\author{
H. SAMELSON \\ Stanford University \\ Stanford, California 94305 \\ RICHARD PIERCE \\ University of Washington \\ Seattle, Washington 98105
}

J. DugundJI

Department of Mathematics University of Southern California Los Angeles, California 90007

BASIL GORDON*

University of California

Los Angeles, California 90024

\section{ASSOCIATE EDITORS}
E. F. BECKENBACH
B. H. NEUMANN
F. WOLE
K. YOSHIDA

\section{SUPPORTING INSTITUTIONS}

\author{
UNIVERSITY OF BRITISH COLUMBIA \\ CALIFORNIA INSTITUTE OF TECHNOLOGY \\ UNIVERSITY OF CALIFORNIA \\ MONTANA STATE UNIVERSITY \\ UNIVERSITY OF NEVADA \\ NEW MEXICO STATE UNIVERSITY \\ OREGON STATE UNIVERSITY \\ UNIVERSITY OF OREGON \\ OSAKA UNIVERSITY \\ UNIVERSITY OF SOUTHERN CALIFORNIA
}

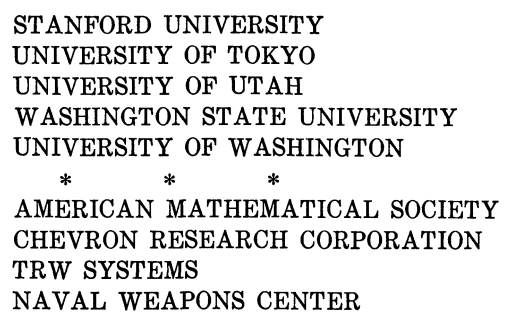

The Supporting Institutions listed above contribute to the cost of publication of this Journal, but they are not owners or publishers and have no responsibility for its content or policies.

Mathematical papers intended for publication in the Pacific Journal of Mathematics should be in typed form or offset-reproduced, (not dittoed), double spaced with large margins. Underline Greek letters in red, German in green, and script in blue. The first paragraph or two must be capable of being used separately as a synopsis of the entire paper. The editorial "we" must not be used in the synopsis, and items of the bibliography should not be cited there unless absolutely necessary, in which case they must be identified by author and Journal, rather than by item number. Manuscripts, in duplicate if possible, may be sent to any one of the four editors. Please classify according to the scheme of Math. Rev. 36, 1539-1546. All other communications to the editors should be addressed to the managing editor, Richard Arens, University of California, Los Angeles, California, 90024.

50 reprints are provided free for each article; additional copies may be obtained at cost in multiples of 50 .

The Pacific Journal of Mathematics is published monthly. Effective with Volume 16 the price per volume (3 numbers) is $\$ 8.00$; single issues, $\$ 3.00$. Special price for current issues to individual faculty members of supporting institutions and to individual members of the American Mathematical Society: $\$ 4.00$ per volume; single issues $\$ 1.50$. Back numbers are available.

Subscriptions, orders for back numbers, and changes of address should be sent to Pacific Journal of Mathematics, 103 Highland Boulevard, Berkeley, California, 94708.

PUBLISHED BY PACIFIC JOURNAL OF MATHEMATICS, A NON-PROFIT CORPORATION

Printed at Kokusai Bunken Insatsusha (International Academic Printing Co., Ltd.), 7-17, Fujimi 2-chome, Chiyoda-ku, Tokyo, Japan.

* Acting Managing Editor. 


\section{Pacific Journal of Mathematics}

\section{Vol. 32, No. $2 \quad$ February, 1970}

Harry P. Allen and Joseph Cooley Ferrar, Jordan algebras and exceptional subalgebras of the exceptional algebra $E_{6} \ldots \ldots \ldots \ldots \ldots \ldots \ldots 283$

David Wilmot Barnette and Branko Grünbaum, Preassigning the shape of a

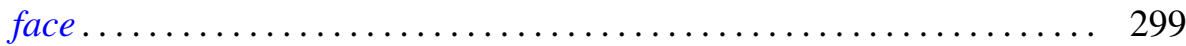

Robert Francis Craggs, Involutions of the 3-sphere which fix 2-spheres . . . . 307

David William Dean, Bor-Luh Lin and Ivan Singer, On k-shrinking and $k$-boundedly complete bases in Banach spaces ................ 323

Martin Engert, Finite dimensional translation invariant subspaces ....... 333

Kenneth Lewis Fields, On the global dimension of residue rings ......... 345

Howard Gorman, The Brandt condition and invertibility of modules ....... 351

Benjamin Rigler Halpern, A characterization of the circle and interval ..... 373

Albert Emerson Hurd, A uniqueness theorem for second order quasilinear

hyperbolic equations ............................... 415

James Frederick Hurley, Composition series in Chevalley algebras ...... 429

Meira Lavie, Disconjugacy of linear differential equations in the complex

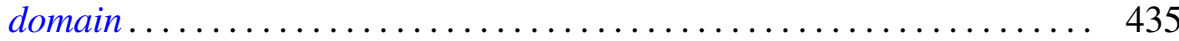

Jimmie Don Lawson, Lattices with no interval homomorphisms ......... 459

Roger McCann, A classification of center-foci ................. 467

Evelyn Rupard McMillan, On continuity conditions for functions . . . . . . . 479

Graciano de Oliveira, A conjecture and some problems on permanents .... 495

David L. Parrott and S. K. Wong, On the Higman-Sims simple group of order $44,352,000$.

Jerome L. Paul, Extending homeomorphisms ................. 517

Thomas Benny Rushing, Unknotting unions of cells .............. 521

Peter Russell, Forms of the affine line and its additive group.......... 527

Niel Shilkret, Non-Archimedean Gelfand theory ................. 541

Alfred Esperanza Tong, Diagonal submatrices of matrix maps.......... 551 